\title{
Producing ultra high energy cosmic rays from AGN magnetic luminosity
}

\author{
Carlos H. Coimbra-Araújo and Rita C. Anjos \\ Departamento de Engenharias e Exatas, Universidade Federal do Paraná, Rua Pioneiro 2153, \\ 85950-000, Palotina, Brazil. \\ email: carlos.coimbra@ufpr.br
}

\begin{abstract}
The present work proposes a method to calculate the AGN magnetic luminosity fraction to be converted into ultra high energy cosmic rays (UHECRs) luminosities for nine UHECR AGN Seyfert sources based on the respective observation of gamma ray upper limits. The motivation for such calculation comes attached to the fact that a fraction of the magnetic luminosity $\left(L_{B}\right)$ produced by Kerr black holes in some AGNs can produce the necessary energy to accelerate UHECRs beyond the GZK limit, observed, e.g., by the Auger experiment. Nevertheless, the direct detection of those UHECRs has a lack of information about the direction of the source from where those cosmic rays are coming, since charged particles are deflected by the intergalactic magnetic field. Such problem arises alternative methods to evaluate the luminosity of UHECRs from the observation of upper limits during the propagation.
\end{abstract}

\section{Introduction}

The nature and the origin of the ultra high energy cosmic rays (UHECRs) with energies higher than $10^{19} \mathrm{eV}$ are subject of many investigations. It is a common sense in the literature about the topic that the main extragalactic candidates for accelerating particles to those highest energies are active galactic nuclei (AGNs) (Henri et al. 1999), the most powerful radiogalaxies (Takahara 1990, Rachen \& Biermann 1993), and also gamma ray bursts, fast spinning newborn pulsars, interacting galaxies, large-scale structure formation shocks and a plenty of other phenomena (Kotera \& Olinto 2011). All the potential of a source to accelerate particles up to the exposed extreme energies is summarized in the well-known Hillas plot (Hillas 1984).

From the point of view of Earth experiments, the deduction of cosmic ray luminosities can possibly shed some light on radiative bounds of UHECR potential sources, as can be seen in Supanitsky \& de Souza (2013) and in Anjos et al. (2014), where it is shown that the methods of UHECR propagation from the source to Earth and the measured upper limit on the integral flux of $\mathrm{GeV}-\mathrm{TeV}$ gamma-rays can infer upper limits of proton and total UHECR (iron) luminosities. This comes from the fact that gamma-rays can be produced as a result of the cosmic ray propagation and contribute to the total flux measured from the source. Cascading background to produce $\mathrm{GeV}-\mathrm{TeV}$ gammas comes from the interaction of UHECRs with the Extragalactic Background Light $(\mathrm{EBL})\left(\approx 10-10^{5} \mathrm{GeV}\right.$ gammas), with the Cosmic Microwave Background $(\mathrm{CMB})\left(\approx 10^{5}-10^{10} \mathrm{GeV}\right)$ and with the radio background $\left(10^{10}-10^{13} \mathrm{GeV}\right)$, see more details in (Blumenthal 1970, Puget et al. 1976, Kelner \& Aharonian 2008, De Angelis et al. 2013). In this way, gammaray observation and respective upper limits are advantageous in comparison to direct UHECR observations, mainly in the aspect of the reconstruction of trajectories to see the main probable candidate sources and respective luminosities in UHECRs.

Following the method summarized above, the present work investigates nine AGNs as UHECR sources (see Table 1). It is calculated upper limits of UHECRs luminosities 
to be compared to the theoretical magnetic/jet luminosity of those AGNs to therefore calculate the $\eta_{C R}$ conversion fraction.

\section{Upper Limits of UHECRs}

The reconstructed direction of a charged cosmic-ray particle might not point back exactly to its source due to deviation in the magnetic fields. Given this limitation, the study of the origin of the cosmic rays is restricted to limited classes of sources and any important information involves the convolution of measured quantities with propagation models and catalogs of objects. On the other hand, gamma-rays are not deviated by magnetic fields. At TeV energies a few hundred gamma-ray sources have been discovered by the past and current Imaging Atmospheric Cherenkov Telescopes (IACTs). Also, high energy gamma rays $\left(10 \mathrm{GeV}<E_{\gamma}<300 \mathrm{GeV}\right)$ have been detected by space and ground instruments, as FERMI-LAT (Adbo et al. 2010), VERITAS (Galante et al. 2009), H.E.S.S (Aharonian et al. 2008) and MAGIC (Antonelli et al. 2010). The method described in Supanitsky \& de Souza (2013) and in Anjos et al. (2014) connects those measured upper limits with the source UHECR cosmic ray luminosity $\left(L_{C R}^{U L}\right)$ by

$$
L_{C R}^{U L}=\frac{4 \pi D_{s}^{2}\left(1+z_{s}\right)\langle E\rangle_{0}}{K_{\gamma} \int_{E_{t h}}^{\infty} d E_{\gamma} P_{\gamma}\left(E_{\gamma}\right)} I_{\gamma}^{U L}\left(>E_{\gamma}^{t h}\right),
$$

where $I_{\gamma}^{U L}\left(>E_{\gamma}^{t h}\right)$ is the upper limit on the integral gamma-ray flux for a given confidence level and energy threshold, $K_{\gamma}$ is the number of gamma rays generated from the cosmic ray particles, $P_{\gamma}\left(E_{\gamma}\right)$ is the energy distribution of the gamma-rays arriving on Earth, $E_{\gamma}$ is the energy of gamma-rays, $\langle E\rangle_{0}$ is the mean energy, $D_{s}$ is the comoving distance and $z_{s}$ is the redshift of the source. This method allows one to calculate upper limits on the proton and total luminosities for energies above $10^{18} \mathrm{eV}$. Also, it illustrates techniques to study the origin of UHECRs from multi-messenger GeV-TeV gamma-rays and it has been used to calculate at least upper limits for thirty sources (AGNs), with redshift smaller than 0.048 and UHECR spectra measured by the Pierre Auger (Schulz et al. 2013) and Telescope Array (TA) (Bergman 2013) observatories. For this same method, CTA Observatory (Actis et al. 2011) will also allow, in the future, a range of new UHECR luminosity calculations.

\section{AGN Magnetic luminosities}

In what follows it is described the production of UHECR luminosities based on the possible relation between magnetic flux accumulation and jet production efficiency. To mimic AGN powering fluxes, let us consider a system with a rotating central $\mathrm{BH}$ that is producing electromagnetic Poynting flux, i.e., it drives magnetic field to accelerate particles via Blandford-Znajek mechanism. The necessary condition to use BlandfordZnajek mechanism (Blandford \& Znajek 1977) is that $\Phi_{d}>\Phi_{B H, \max }(\dot{M})$, i. e., the net poloidal magnetic flux $\Phi_{d}$ trapped in the disk is larger than the maximum that can be confined on the $\mathrm{BH}$ caused by pressure of the accreting plasma. Satisfied this condition, the rate of energy extraction from the rotating $\mathrm{BH}$ via the Blandford-Znajek (BZ) mechanism yields the magnetic luminosity (see Coimbra-Araújo \& Anjos (2015)). As AGN jets can be the main extragalactic sources of UHECRs, one can write the UHECR luminosity $L_{C R}^{T h e o r y}$ as a fraction $\eta$ of the magnetic luminosity:

$$
L_{C R}^{\text {Theory }}=\eta L_{B} .
$$




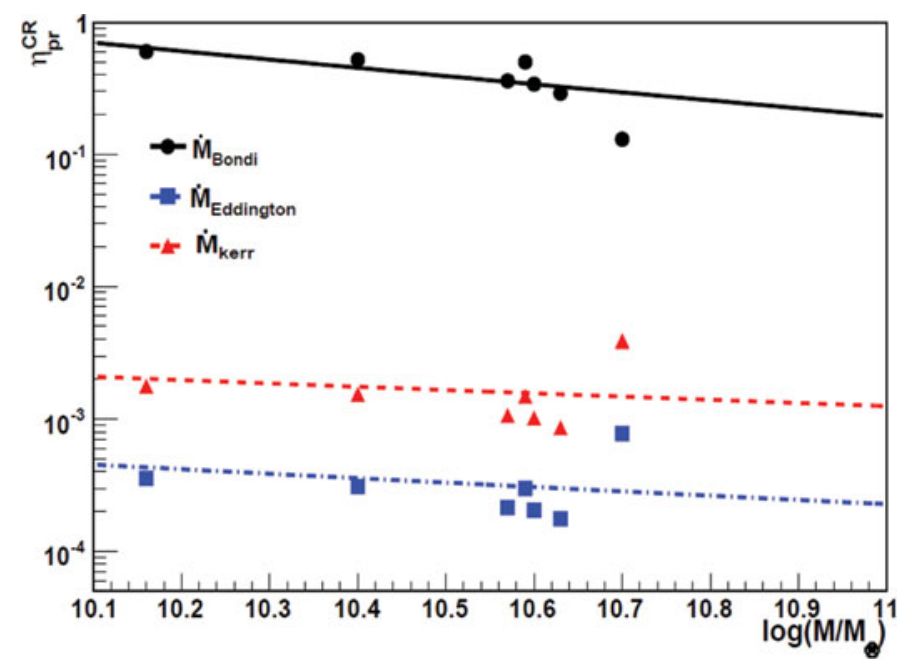

Figure 1. Production of UHECRs in AGNs with Kerr black holes (axisymmetric accretion by Kerr black holes). In comparison we show the limits using the Eddington and Bondi accretion rates.

Table 1. Comparison between cosmic ray luminosity $L_{p r}^{U L}$ (protons) from the method derived from eq. (2.1) and the theoretically calculated range $L_{C R \text { Tin }}^{\text {Theory }}-L_{C R \text { max }}^{\text {Theory }}$ of cosmic ray luminosities, from nine sources. Here it is considered the case for $a=0.7$.

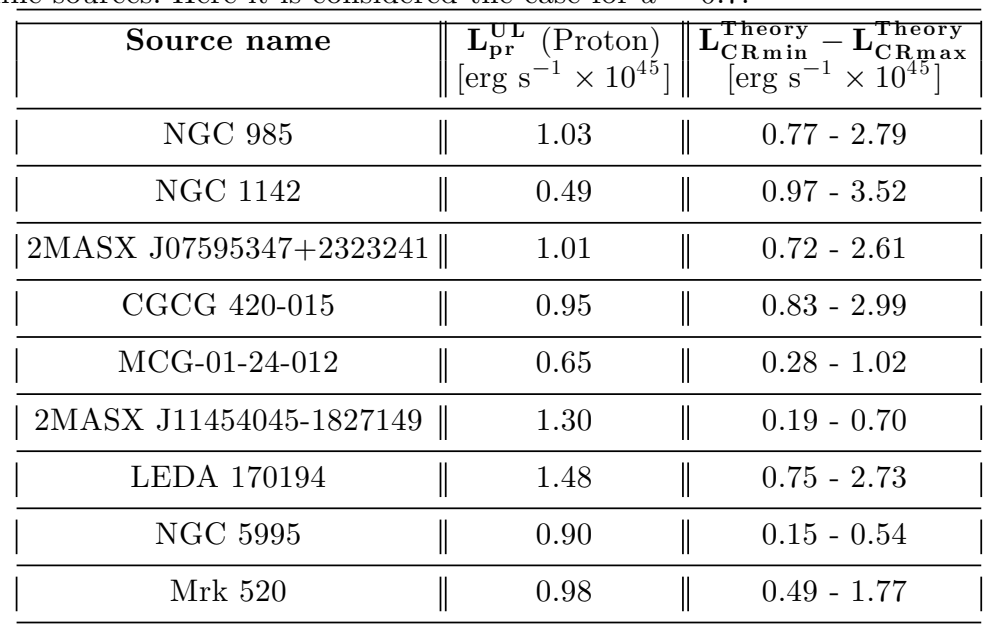

For example, bounds on the fraction $\eta_{p r}$ of $L_{B}$ to be converted in relativistic protons, from upper limits as a function of $\mathrm{BH}$ spin $a$ is written as

$$
\eta(a)_{p r}=\frac{4 \pi D_{s}^{2}\left(1+z_{s}\right)}{10(\phi / 50) x_{a}^{2} f_{a}\left(x_{a}\right) \dot{M} c^{2}} \frac{\langle E\rangle_{0} I_{\gamma}^{U L}\left(>E_{\gamma}^{t h}\right)}{K_{\gamma} \int_{E_{t h}}^{\infty} d E_{\gamma} P_{\gamma}\left(E_{\gamma}\right)},
$$

where $\phi$ and $f\left(x_{a}\right)$ are BZ functions described in McKinney \& Tchekhovskoy (2012). For the spin range $0.44<a<0.8$ where most black holes are expected to lie, the fraction $\eta_{p r}$ varies from $\sim 5 \%$ to $50 \%$ for $a=0.45$ and from $2 \%$ to $10 \%$ for $a=0.8$ Anjos et al. (2016), Coimbra-Araújo \& Anjos (2015). Such limits are calculated with the aid of Table 
1, which shows nine sources with their respective calculated upper limits in UHECR luminosity for protons.

\section{Results and discussion}

Fig. 1 shows how Kerr black holes, using a axisymmetric accretion with a central spinning hole, according to AGN masses, will produce UHECRs. A $M^{-5.8}$ power law is obtained, according to the AGN mass. The interplay between $L_{C R}^{\text {Theory }}$ upper limits and the magnetic luminosity $L_{B}$ is explicitly investigated here by a conversion rate $\eta_{C} R$ calculated here and developed in Coimbra-Araújo \& Anjos (2015) and Anjos et al. (2016). The comparison among $L_{C R}^{\text {Theory }}$, the upper limit $L_{p r}$ and $L_{B}$ offer limits to the conversion fraction $\eta_{p r}$ for each source. Here, the accretion mechanism has spherical symmetry (Bondi mechanism) and also axial symmetry (Fig. 1), where it is assumed the general case of a Randall-Sundrum-Kerr central black hole, see Coimbra-Araújo \& Anjos (2015).

\section{References}

Abdo, A. A., Ackermann, M., Ajello, M., Allafort, A., Antolini, E., Atwood, W., Axelsson, M., Baldini, L., Ballet, J., Barbiellini, G., et al., The Astrophysical Journal 715, p. 429 (2010).

Actis, M., Agnetta, G., Aharonian, F., Akhperjanian, A., Aleksic, J., Aliu, E., Allan, D., Allekotte, I., Antico, F., Antonelli, L., et al., Experimental Astronomy 32, 193-316 (2011).

Aharonian, F., Akhperjanian, A., De Almeida, U. B., Bazer-Bachi, A., Behera, B., Beilicke, M., Benbow, W., Bernlohr, K., Boisson, C., Bolz, O., et al., Astronomy 83 Astrophysics 478, 387-393 (2008).

Anjos, R., De Souza, V., \& Supanitsky, A., Journal of Cosmology and Astroparticle Physics 2014, p. 049 (2014).

Anjos, R. C., Coimbra-Araújo, C. H., da Rocha, R., \& de Souza, V., Journal of Cosmology and Astroparticle Physics 2016, p. 014 (2016).

Antonelli, L., Antoranz, P., Backes, M., Barrio, J., Bastieri, D., Bednarek, W., Berdyugin, A., Berger, K., Bernardini, E., Biland, A., et al., The Astrophysical Journal Letters 723, p. L207 (2010).

Bergman, D., "Ta spectrum summary," Proceedings of the 33rd International Cosmic Ray Conference, Rio de Janeiro, Brazil (2013).

Blandford, R. D. \& Znajek, R. L., Monthly Notices of the Royal Astronomical Society 179, 433-456 (1977).

Blumenthal, G. R., Physical review D 1, p. 1596 (1970).

Coimbra-Araújo, C. \& Anjos, R., Physical Review D92, p. 103001 (2015).

De Angelis, A., Galanti, G., \& Roncadelli, M., Monthly Notices of the Royal Astronomical Society 432, 3245-3249 (2013).

Dutan, I. \& Caramete, L. I., Astroparticle Physics 62, 206-216 (2015).

Galante, N., et al., arXiv preprint arXiv:0912.3850 (2009).

Henri, G., Pelletier, G., Petrucci, P.-O., \& Renaud, N., Astroparticle Physics 11(3) , 347-356 (1999).

Hillas, A., Annual review of astronomy and astrophysics 22, 425-444 (1984).

Kelner, S. \& Aharonian, F., Physical Review D78, p. 034013 (2008).

Kotera, K. \& Olinto, A. V., Annual Review of Astronomy and Astrophysics 49, 119-153 (2011).

McKinney, J. C., Tchekhovskoy, A., \& Blandford, R. D., Monthly Notices of the Royal Astronomical Society 423, 3083-3117 (2012).

Puget, J., Stecker, F., \& Bredekamp, J., The Astrophysical Journal 205, 638-654 (1976).

Rachen, J. P. \& Biermann, P. L., Astronomy and Astrophysics 272, p. 161 (1993).

Schulz, A., et al., Proceedings of the 33rd ICRC, Rio de Janeiro, Brasil (2013).

Supanitsky, A. \& de Souza, V., Journal of Cosmology and Astroparticle Physics 2013, p. 023 (2013).

Takahara, F., Progress of Theoretical Physics 83(6), 1071-1075 (1990). 